\title{
Association of Cancer and Anticancer Therapy with Stress Cardiomyopathy
}

\author{
HAO SUN, Y. JI, S. LI AND H. DONG*
}

Department of Cardiovascular Medicine, Dezhou People's Hospital, No. 1751 Xinhu Street, Dezhou City, Shandong Province, 253000, China

Hao et al.: Anticancer therapy with stress cardiomyopathy

\begin{abstract}
Cancer, a chronic malignancy is the ultimately cause of stress cardiomyopathy since its features are expressively precipitated by cancer-mediated physical and emotional stress. Just like an acute coronary disease or acute heart failure, stress cardiomyopathy or Takotsubo cardiomyopathy, is a clinical condition presenting severe chest pain as a major symptom. Physical, emotional or medical stress initiates the disorder with a condition of severe left ventricular systolic dysfunction. The emotional stress or anxiety associated with cancer malignancy diagnosis, prevailing stage of cancer itself and the physical stresses induced by surgical, radiation and systemic antineoplastic treatment of cancer are the major factors accelerating stress cardiomyopathy. Stress cardiomyopathy is progressively increasing in cancer patients and is an adverse consequence in such patients. In the current report, the stress syndrome being identified in cancer patients after systemic antineoplastic therapy has been reviewed. In the current report, information assembled and examined include clinical symptoms, electrocardiogram, laboratory information, transthoracic echocardiogram, coronary angiogram outcomes and patient consequences. Cancer patients are at amplified risk of developing stress syndrome/stress cardiomyopathy and it is extremely imperative to investigate the drugs involved in the progression of stress syndrome.
\end{abstract}

Key words: Chemotherapy, stress cardiomyopathy, cancer

Co-existence of cardiovascular diseases has been observed in cancer patients taking systemic chemotherapy. Sometimes, differentiation among chemotherapy-induced cardio toxicity and cardiac disorders occurring from any other cause (not associated to cancer therapy) are extremely challenging for healthcare personals. Identification of chemotherapyprovoked cardio toxicity is highly significant since the continued therapy with such medication may cause irreversible cardiac damage. However, morbidity and mortality rate of cancer patients may be dramatically increased attributed to the earlier discontinuation of an efficacious antineoplastic medicine/drug upon appearance of concurrent cardiac conditions not directly associated to chemotherapy.

Stress cardiomyopathy (SCM) may be described as a single cardiac event legitimately caused by chemotherapy or might be completely unconnected to it. SCM refers to a clinical condition characterized by severe left ventricular (LV) systolic dysfunction occurring after abrupt exposure to physical, medical

*Address for correspondence

E-mail: sddhw@sina.cn or emotional stress and is primarily indicated by acute chest pain that can mimic acute coronary syndrome (ACS) or an acute heart failure. In contrast to ACS, SCM patients display normal coronary angiogram and reversible dysfunction of left ventricle that exceeds beyond epicardial coronary distribution with most of the cases being fully recovered within couple of days or weeks. Transient akinesis and systolic apical ballooning of LV occurs with the cardiac basal segments being hyperdynamic ${ }^{[1]}$. Although the disease pathophysiology is not well defined however, coronary microvascular disease, increased catecholamine contents and vasoconstriction of coronary artery are considered to be the vital mediating mechanisms ${ }^{[2]}$. Different stressors particularly emotional and psychological stress, drugs/ medicines, infection, surgery and aggravation of chronic illnesses may stimulate such mechanisms ${ }^{[3]}$. Likewise, for SCM, hereditary/genetic preferences have also been reported suggesting the disease prevalence in individuals with familial case ${ }^{[4]}$. Currently certain anticancer drugs have been observed to stimulate SCM progression. Although the cardiotoxicity of chemotherapeutic drugs 
has been discussed in numerous reports, however the information on SCM caused by antineoplastic therapy is meagre. Until now no well-known correlation between a dose of chemotherapeutic agents and SCM has been reported. Conceivable etiologic connections between chemotherapeutic agents and SCM have been explored in this review. Both traditional and the latest targeted cancer therapies including immune checkpoint inhibitors have been reviewed. Surely, current review would reveal the correlation between SCM and cancer as well as SCM and chemotherapeutic drugs.

\section{Stress cardiomyopathy (SCM):}

Dote and colleagues, described SCM for the first time in Japan ${ }^{[1]}$. SCM was referred to as "Takotsubo" based on specific LV (apical and mid segments) ballooning that resembled Japanese octopus trap pot in shape. SCM is often termed as apical ballooning syndrome, broken heart syndrome, myocardial stunning and stress cardiomyopathy. The intense stressors of SCM include emotional and mental stress. SCM predominantly influences post-menopausal women $(>90 \%)$ based on their frequent exposure to unexpected stressors. Patients suffering from SCM exhibit clinical symptoms that fluctuate greatly from chest pain $(63 \%)$ to exertionbased dyspnea $(8 \%)$ and syncope $(3 \%)^{[3]}$. Symptomatic fluctuation creates additional diagnostic challenges since such symptom constellation could mimic cerebrovascular illnesses, myocardial infarction (MI) and pulmonary embolism. Upon electrocardiographic investigation, SCM patients present uneven ECG alterations primarily the inversion of $\mathrm{T}$ wave and elevation of ST segment ${ }^{[5]}$. T wave inversion and ST segment elevation has been illustrated in around 30.4$34.2 \%$ of cases whereas non-specific ST-T wave variations were observed in $35.2 \%$ cases, respectively ${ }^{[5]}$. Slightly elevated levels of cardiac enzymes have been provoked in SCM patients; however the elevation degree is extremely lower as compared to the myocardial area being affected. Angiographic examination indicated no evidence of obstructive coronary artery disease (CAD, indicated by $>50 \%$ constriction of coronary artery $)^{[2]}$.
Imaging techniques included left ventriculography and echocardiography represented transient LV dysfunction and ballooning of LV segment. Three distinctive LV contraction irregularities have been observed in SCM patients. The most familiar kind included apical akinesis exploiting hyperkinesis of basal segments. The other less familiar examples encompass combined akinesis (mid-LV and apical involvement), solitary akinesis of mid-LV segment and solitary basal akinesis ${ }^{[6]}$.

Extreme left ventricular ejection fraction (LVEF) reduction has been noticed in SCM individuals with LVEF value ranged between $15-30 \%$ (mean value $~ 20$ $\%$ ) as compared to the normal subjects exhibiting LVEF value between $55-75 \%{ }^{[2]}$. In most of subjects, regular follow up may restore LVEF value higher than $50 \%$. Although most of the patients exhibit cardiac recovery within 4-6 w however, in few patients extended duration of 2.5 mo to $1 \mathrm{y}$ was required for the regularization of LV systolic function ${ }^{[2,7]}$. The extended time duration needed for LV systolic function regularization may augment the risk of emboli formation or embolism. Rarely SCM may result in certain complication like arrhythmias (atrial or ventricular), hemodynamic instability, symptoms of heart failure and cardiogenic shock and ultimate death. To assist SCM diagnosis, Mayo Clinic diagnostic criteria recommended by the Mayo Clinic in 2004 can be utilized ${ }^{[8]}$. Treatment practices encompass concomitant administration ACE inhibitors and $\beta$-blockers; medications that limit the catecholamine activation and profoundly restrict one of major pathogenic pathways. In hospitals, the mortality rate higher than $16 \%$ has been reported in SCM patients ${ }^{[9]}$. Due to the lack of extended follow up considerations, information pertaining to long-term prognosis and subsequent consequences is unavailable.

\section{Induction mode of SCM:}

Though the pathophysiologic basis of SCM progression is accurately defined however, several proposed mechanisms include abundant catecholamine secretion, vasoconstriction or vasospasm of coronary arteries, microvascular syndrome and overexpression of some

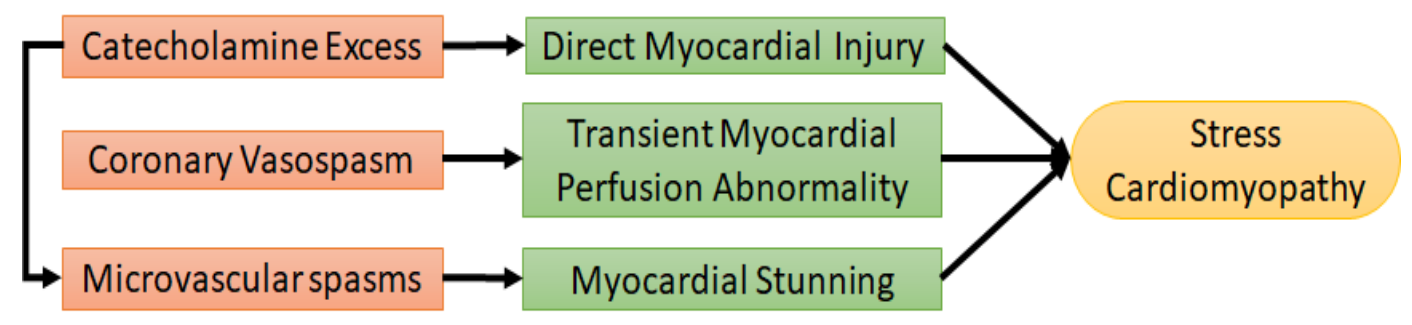

Fig. 1: Induction mode of stress cardiomyopathy 
cardiac genes (fig. 1). Catecholamine discharge during stressful conditions such as physical, emotional or mental stress play a vital role in the progression of SCM. According to Wittstein et al. patients with LV dysfunction exhibited elevated catecholamine contents during or after emotional stress ${ }^{[2]}$. Similar findings were also reported by Abraham et al. which greatly supported the fundamental role of catecholamines in precipitating SCM. Abraham et al. observed immediate acceleration of SCM features after intravenous administration of dopamine or norepinephrine infusion ${ }^{[10]}$.

cAMP stimulation elevates the intracellular contents of norepinephrine within cardiac myocytes with ultimate cardiac impairment. $\beta$-Adrenergic blocker utilization demotes cardiac impairment and such findings positively favour norepinephrine's pivotal role in SCM precipitation. Coronary artery vasospasm, the other primary mechanism involved in the precipitation of SCM was favoured by the findings of multifocal coronary vasospasm and transient myocardial perfusion abnormalities. Medicines also induce SCM and druginduced SCM would be particularly considered in patient with no clear recognition of emotional or other stress triggers ${ }^{[11]}$.

\section{Association between cancer and SCM:}

The psychological, emotional or physical stressful conditions created by cancer, a chronic illness considerably intensifies the risk of SCM progression. In cancer patients, emotional and physical stressors act as inducers of SCM. Cancer diagnosis or identification of its particular inflammatory state initiates emotional disquietude while cancer chemotherapy initiates physical stress in patients (fig. 2) ${ }^{[12,13]}$. Further, it has been postulated that various paraneoplastic negotiators circulating in the blood may stimulate adrenergic receptors of cardiac tissues leading to the impairment contractile function. Indeed, oncologists and cardiologists are completely aware of chemotherapeutic drug (such as trastuzumab and anthracyclines)-induced cardiotoxicity ${ }^{[14]}$. Cardiac arrhythmias, hypertension, ischemia, myocarditis, progressive atherosclerosis, QT prolongation, venous thromboembolism and ventricular dysfunction are the numerous myocardial adverse effects caused by cancer treatment ${ }^{[15-17]}$. 5-Fluorouracil (5-FU), a renowned anticancer drug intensely implicates SCM precipitation. Antiangiogenics, capecitabine, combretastatin, rituximab, taxols, vascular endothelial growth factors inhibitors and several other chemotherapeutic drugs are known to cause cardiotixicity (Tables 1 and 2). SCM is probably an under-recognized and thus under-diagnosed disease entity among cancer patients receiving chemotherapy ${ }^{[12]}$. Perhaps SCM is not appropriately identified and diagnosed in cancer subjects taking anticancer drugs. PubMed database was explored using key terms, stress cardiomyopathy, cancer, and antineoplastic therapy to study cancer patients taking systemic anticancer medications and exhibiting symptoms of SCM. The acquired and evaluated data included patient outcomes along with clinical manifestations (signs and symptoms of disorder), diagnostic information, findings of electrocardiogram (ECG), transthoracic echocardiogram and coronary angiogram. Assessment of anticancer drugs reported to initiate SCM prognosis is the information required to be explored.

\section{ASSOCIATION BETWEEN CANCER CHEMOTHERAPY AND SCM}

\section{5-FU:}

5-FU belongs to the antimetabolites class of antineoplastic agents. A pyrimidine analogue acting via inhibition of DNA synthesis. In malignant cells, its mechanism of action is through inhibition of thymidylate synthase with subsequent interruption of DNA synthesis and ultimate cell death ${ }^{[22]}$. 5-FU is most frequently prescribed as an intravenous bolus injection or infusion for the cytostatic management of solid tumors encompassing bladder, breast, colorectal, esophageal, gastric, pancreatic and prostate cancers. During colorectal cancer therapy, 5-FU contributes

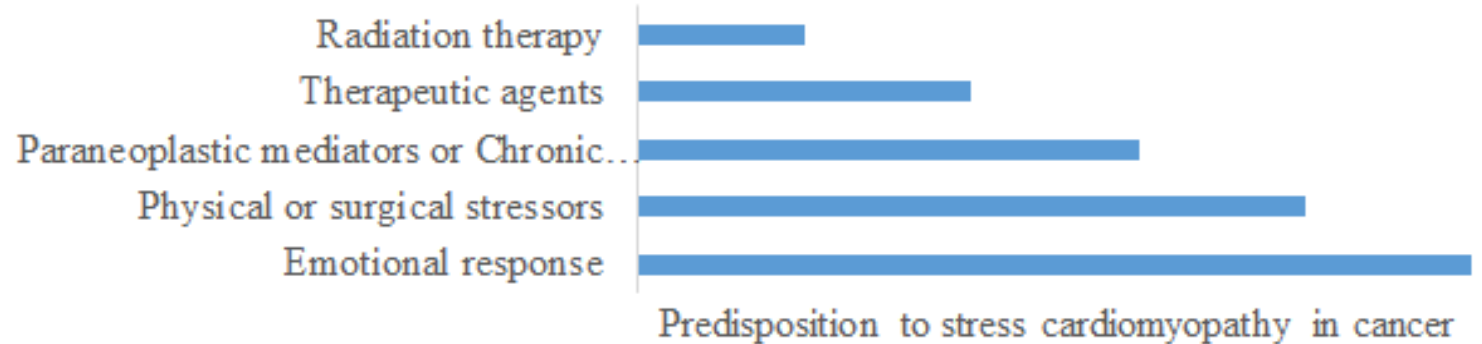

Fig. 2: Cancer and chemotherapy predisposes SCM 
TABLE 1: RETROSPECTIVE DATA ON AGE, CANCER TYPE AND CHEMOTHERAPY USED AND THE RESULTING SCM STATUS

Patient, disease and treatment

Salient findings

References

60 y old female with colorectal cancer (stage III) First cycle of chemotherapy was accompanied by SCM with

treated with 5-FU infusion chest pain, sinus tachycardia, and troponin- $1=0.38$

62 y old female with rectal adenocarcinoma treated Sixth cycle of chemotherapy was accompanied by SCM with 5-FU + levofolinate with chest pain, sinus tachycardia, and troponin- $1=0.82$

58 y old female with metastatic adenocarcinoma First cycle of chemotherapy was accompanied by SCM with

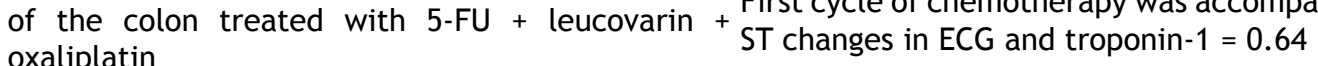

oxaliplatin

52 y old male with squamous cell carcinoma of soft First cycle of chemotherapy was accompanied by SCM with palate treated with $5-\mathrm{FU}+$ cisplatin sinus tachycardia

54 y old male with metastatic adenocarcinoma First cycle of chemotherapy was accompanied by SCM of the colon treated with 5-FU + leucovarin + with chest pain, lateral STE with reciprocal changes with oxaliplatin intermittent LBBB in ECG, and troponin-1 = 4.07

48 y old male with adenocarcinoma of the colon First cycle of chemotherapy was accompanied by SCM treated with $5-\mathrm{FU}+$ leucovarin + oxaliplatin with chest pain, apicolateral STD in ECG and TWI, and troponin-1 $=0.5$

$59 \mathrm{y}$ old male with invasive adenocarcinoma of

sigmoid colon treated with 5-FU + leucovarin + First cycle of chemotherapy was accompanied by SCM with oxaliplatin

chest pain, upsloping STE in ECG, and troponin-1 = 1.0

48 y old male with gastric adenocarcinoma treated Seventh cycle of chemotherapy was accompanied by SCM with 5-FU + docetaxel + cisplatin with sinus tachycardia and troponin-1 $=2.87$

14 y old male with metastatic nasopharyngeal First cycle of chemotherapy was accompanied by SCM with carcinoma treated with 5-FU infusion + cisplatin sinus tachycardia

79 y old female with metastatic adenocarcinoma of Tenth cycle of chemotherapy was accompanied by SCM the colon treated with 5-FU infusion with chest pain, STE in ECG, and troponin-1 $=1.06$

81 y old female with metastatic adenocarcinoma Fifth cycle of chemotherapy was accompanied by SCM with of the colon treated with 5-FU + folinic acid + chest pain, TWE in various leads in ECG, and troponin-1 = capecitabine 0.35

$47 \mathrm{y}$ old female with metastatic invasive ductal First cycle of chemotherapy was accompanied by SCM with breast carcinoma treated with capecitabine chest pain, STE in ECG, and troponin-1 $=0.19$

$55 \mathrm{y}$ old male with adenocarcinoma of the colon First cycle of chemotherapy was accompanied by SCM with treated with capecitabine chest pain, STE in ECG, and HS troponin $=89$

62 y old male with adenocarcinoma of the colon First cycle of chemotherapy was accompanied by SCM with treated with capecitabine STE in ECG

39 y old female with inflammatory breast cancer First cycle of chemotherapy was accompanied by SCM with treated with capecitabine sinus tachycardia and troponin-1 $=0.34$

76 y old male with colon cancer treated with First cycle of chemotherapy was accompanied by SCM with bevacizumab STE in ECG

61 y old male with non-small cell lung cancer Second cycle of chemotherapy was accompanied by SCM treated with bevacizumab with sinus tachycardia and troponin-1 $=2.5$

71 y old female with anaplastic thyroid cancer treated First cycle of chemotherapy was accompanied by SCM with with doxorubicin + cisplatin + Combretastatin TWI in I, aVL, V2-V5 in ECG and troponin-1 = 0.85

78 y old female patient with anaplastic thyroid First cycle of chemotherapy was accompanied by SCM with cancer treated with Doxorubicin + Cisplatin + + deep symmetric TWI in ECG
combretastatin

$66 \mathrm{y}$ old male with CLL treated with First cycle of chemotherapy was accompanied by SCM with methylprednisolons + rituximab sinus tachycardia and troponin-1 $=0.14$

57 y old female with renal cell carcinoma treated First cycle of chemotherapy was accompanied by SCM with with Sunitinib chest pain, STE, and troponin-1 = 1.2

71 y old female with renal cell carcinoma treated First cycle of chemotherapy was accompanied by SCM with axitinib with chest pain, anterolateral STE in ECG, and troponin-1 $=6.95$

50 y old female with metastatic invasive ductal Thirteenth cycle of chemotherapy was accompanied by breast treated with carboplatin + docetaxel + SCM with chest pain, TWI in V1-2 in ECG, and troponin-1 trasruzumab $\quad=0.15$

55 y old male with non M3-AML treated with Eleventh cycle of chemotherapy was accompanied by SCM daunorubicin + cytarabine with chest pain, sinus tachycardia, and troponin-1 $=38.64$ 83 y old female with metastatic melanoma treated First cycle of chemotherapy was accompanied by SCM with with Ipilimumab chest pain, sinus tachycardia, and troponin- $1=0.98$ 
TABLE 2: IMPROVEMENT IN LVEF (\%), CARDIOLOGICAL FINDING, AND FINAL OUTCOME

\begin{tabular}{lcc}
\hline Improvement in LVEF (\%) and final outcome & Cardiological finding & References \\
\hline From $15-20 \%$ to $55-60 \%$, patient survived & Global hypokinesis; Apical ballooning; Non-obstructive CAD & {$[18]$} \\
From $28 \%$ to $67 \%$, patient survived & Apical akinesis; Normal coronary arteries & {$[19]$} \\
From $15 \%$ to $45 \%$, patient survived & Diffused hypokinesis & {$[20]$} \\
From $20 \%$ to $60 \%$, patient survived & & {$[21]$} \\
From $30 \%$ to $65 \%$, patient survived & Normal coronary arteries & {$[17]$} \\
From $15 \%$ to $30 \%$, patient survived & Global hypokinesis & {$[22]$} \\
From $10 \%$ to $68 \%$, patient survived & Global hypokinesis; Non-obstructive CAD & {$[23]$} \\
From $15 \%$ to $50 \%$, patient deceased & Hypokinesis; Non-obstructive CAD & {$[24$} \\
From $20 \%$ to EF recovered, patient deceased & & $[25]]$ \\
From $34 \%$ to $70 \%$, patient survived & Apical akinesis; Non-obstructive CAD & {$[20,26]$} \\
From $35 \%$ to $60 \%$, patient survived & Anteroapical wall motion abnormality; Non-obstructive & {$[27]$} \\
From $30 \%$ to $55 \%$, patient survived & CAD & {$[28]$} \\
From $15-20 \%$ to $55 \%$, patient survived & Hypokinesis; Atheromatous changes & {$[29]$} \\
From $35 \%$ to $55-65 \%$, patient survived & Hypokinesis; Non-obstructive CAD & {$[30]$} \\
From $28 \%$ to $62 \%$, patient survived & & {$[31]$} \\
Not applicable, patient survived & Apical kinesis & {$[31]$} \\
From $40-50 \%$ to $55-65 \%$, patient survived & Non-obstructive CAD & {$[32]$} \\
From $50-55 \%$ to $60-65 \%$, patient deceased & Apical akinesis; Non-obstructive CAD & {$[33]$} \\
From $40 \%$ to EF recovered, patient survived & Hypokinesis; Non-obstructive CAD & {$[34]$} \\
From $15-20 \%$ to $68 \%$, patient survived & Global hypokinesis; Non-obstructive CAD & {$[35]$} \\
From $20-25 \%$ to $50-55 \%$, patient survived & Global hypokinesis; Non-obstructive CAD & {$[36]$} \\
Not applicable, patient survived & Systolic bulge; Normal coronary arteries & {$[37]$} \\
From $30-35 \%$ to $50 \%$, patient survived & Hypokinesis; Normal coronary arteries & {$[38]$} \\
\hline
\end{tabular}

to adjuvant chemotherapy and is administered as a combination therapy along with irinotecan or oxaliplatin coupled to leucovorin. The designed combination therapy includes FOLFIRI (5-FU, folinic acid and Irinotecan) or FOLFOX (5-FU, folinic acid and oxaliplatin) regimens ${ }^{[39,40]}$.

Chest pain inclusive or exclusive of electrocardiographic evidence is the most familiar 5-FU-related CV adverse effect. The range of occurrence of 5-FU-centered cardiotoxicity is $1.5-18 \%{ }^{[41]}$. Acute MI accompanying chest pain/angina pain, cardiac arrhythmias, abnormal/ unusual ECG, cardiac failure are the signs and symptoms presented by SCM patients together with some other clinical presentations identifying stress syndrome ${ }^{[21]}$. Probability calculation assessing the likelihood of correlation between administered drug and adverse effects score 8 was determined by Basselin et al. using Naranjo adverse drug reaction probability scale. Basselin et al. narrated pivotal relationship between 5-FU therapy and SCM based on the calculated score ${ }^{[18]}$. In case of 5-FU-induced SCM, the disease persists for prolonged duration of about a month with resultant treatment cessation ${ }^{[24,25]}$. Up till now 10 articles reporting 5-FU-induced/triggered stress syndrome and SCM have been published ${ }^{[23,26,42-44]}$. The patients with age limit of 14 to $79 \mathrm{y}$ were reported in the case studies $^{[45,46]}$. Despite the fact that SCM primarily affects postmenopausal women, comparable incidence of 5-FU-mediated stress syndrome was noticed in both sexes. Most of the patients followed displayed colorectal cancer except two cases with head and neck cancer. 5-FU was administered as a component of primary chemotherapy regimen (FOLFOX, FOLFIRI or cisplatin-based regimen) in all the SCM reported cases. In most of the reported case, clinical manifestations of stress syndrome appeared during or promptly after the course of drug (5-FU continuous infusion) administration. Out of the 10 reported cases, 7 cases depicted SCM incidence during the first round. Chest pain/angina was the clinical presentation observed in majority of patients, however one patient presented ventricular fibrillation (or arrhythmias) related cardiac arrest but remained alive afterwards. During the 5th round of 5-FU infusion another patient presented abrupt circulatory collapse leading to death, although the patient recovered from stress syndrome during the initial round ${ }^{[42,43]}$. All the 10 patients had electrocardiographic abnormalities of variable degree with extremely reduced LVEF interquartile range i.e. $10-35 \%$. Cardiac angiogram/coronary angiography indicated that obstructive CAD was not developed in any patient ${ }^{[47,48]}$. Retrieved myocardial functioning 
was detected in all the patients (except one patient who expired) with LVEF stabilization occurred in a time span of 1 to $4 \mathrm{w}$. A major challenging issue under consideration was the reinstatement of 5-FU therapy subsequent to these cardiotoxic manifestations. A task of 5-FU reinstatement was successfully achieved, yet a $13 \%$ death rate was detected with reintroduction ${ }^{[49]}$. An alternative study reported by Segalini et al. revealed that reinstatement of 5-FU therapy particularly in patients already affected with cardiac toxicity, could result in further cardiac complications particularly cardiopathy (severe angina along with $\mathrm{MI})^{[50]}$. According to their findings, out of 20 patients receiving 5-FU retreatment; 18 patients presented cadio toxic manifestations with 3 patients experienced MI while 2 patients failed to survive the course of retreatment. Hence the available literature does not encourages the reinstatement of 5-FU therapy after restoration of cardiac function; however based on patient requirement the treatment protocols may be designed considering the complete risk-benefit assessment information, vast patientphysicians conversation, and team work implying health care professionals from various disciplines ${ }^{[50]}$.

\section{Capecitabine:}

Capecitabine is an orally administered antineoplastic medication and is classified as an antimetabolite. Capecitabine is used an alternative to 5-FU since it is enzymatically activated to 5-FU within cancer cells ${ }^{[51]}$. Its Initial introduction in 1990 provoked an anticipation that capecitabine would provide promising efficacy and toxicitybalancebased on its distinctive features including preferable activation to 5-FU within the tumor tissues and administration via oral route ${ }^{[52]}$. From the beginning, wide range of cancers including breast, colorectal, esophageal, gastric and many other gastrointestinal tumors has been treated with capecitabine ${ }^{[53]}$. The cardio toxic manifestations of capecitabine are now recognized to be almost similar to 5-FU that obliterated all the previous beliefs. A total of $3 \%$ incidence of cardiaotoxicity was observed in a retrospective study involving 1189 patients treated either with capecitabine or 5-FU. Almost equivalent cardiotoxicity occurrence rate was noticed in both the capecitabine-treated and 5 -FU treated groups ${ }^{[20]}$. Angina, cardiac shock, MI and sudden cardiac arrest were the clinical presentations induced by capecitabine therapy ${ }^{[54]}$. Noteworthy that all the patients on capecitabine therapy displayed SCM clinical manifestations within $72 \mathrm{~h}$, but recovered within $6 \mathrm{w}$ after therapy termination. Just like 5-FU, vasoconstriction of coronary artery/coronary vasospasm is considered to be the most common cardiac side effect associated to capecitabine therapy, however, a rare postulate includes thrombogenesis, myocardial injury and autoimmune disorders ${ }^{[5]}$. Till now the reported literature identified 5 cases of capecitabine-related SCM. The disease affected patients with age limit of 4781 y with equivalent incidence rate in both sexes. None of the patients had previous history of CAD and were taking therapy for colorectal cancer ( 3 cases) $)^{[20,28,29]}$ or metastatic breast cancer ( 2 cases) management ${ }^{[27,30]}$. All cases were found to present SCM features during primary round of capecitabine therapy except a single case being switched to capecitabine after initial treatment with 5 cycles of 5-FU. Abnormal ECG, raised cardiac enzymes, normal coronary angiogram were the clinical manifestations observed in all the capecitabineinduced SCM patients. Complete LVEF restoration occurred within a month (1-4 w).

\section{Bevacizumab:}

Bevacizumab is an antiVEGF humanized monoclonal antibody exerting anticancer or antiangiogenic activity via binding to and the subsequent inhibition VEGF signaling ${ }^{[56]}$. The pivotal role of VEGF in tumor growth, angiogenesis, proliferation and metastasis is well described. Generally it is supposed to promote the homeostasis and overall cardiac functioning of a healthy adult cardiovascular system ${ }^{[57]}$. The anticancer activity of bevacizumab is initiated via multiple mechanisms with the most common one being, inhibition of angiogenesis (neutralization of VEGF-A), lowering of interstitial pressure of cancer cells and promoting delivery of chemotherapeutic moieties. Bevacizumab is the latest antiangiogenic moiety approved by FDA to be administered in combination therapy along with other anticancer drugs for the management of some specific types of tumors including breast cancer, non-small cell lung cancer and metastatic colorectal cancer $^{[58]}$. Cadiotoxic manifestations especially arterial thromboembolism has been related to Bevacizumab ${ }^{[59]}$. Anginal chest pain, elevated blood pressure, MI, transient ischemic attack and stroke are the other adverse effects linked to bevacizumab treatment ${ }^{[60]}$.

Altered or decreased contractile functioning, ventriculomegaly i.e. dilated ventricles and consequent cardiac failure was observed in studies conducted on genetically engineered mouse models exhibiting capability of inhibiting the VEGF signalling pathway ${ }^{[31]}$. Moreover, it is also suggested that cardiac toxicity is provoked by elevated concentration of nanomotors/ chemoattractant particles and inflammatory cytokines 
present within the cardiac tissue and plasma ${ }^{[61]}$. Till now 2 cases of bevacizumab-related SCM have been described ${ }^{[60]}$. First reported case was a male patient of 76 y age with colon cancer experienced ST segment elevation in the inferior and interior subsequently after $48 \mathrm{~h}$ of receiving bevacizumab treatment. The patient presented reduced LVEF wall motion irregularities and absent signal for obstructive CAD during coronary angiography. The second reported case was male patient of $61 \mathrm{y}$ age with non-small cell lung cancer depicted new Q waves (inferior), elevated ST segment and precordial leads upon ECG analysis. Normal coronary angiogram and LV systolic dysfunction indicated bevacizumab-induced SCM. Both the cases remained alive with absolute restoration of LV systolic function within 2 to $3 \mathrm{w}^{[60]}$.

\section{Combretastatin:}

Combretastatin was initially obtained from the African bush willow, Combretum caffrum with several synthetic analogues being developed. It is comprised of combretastatin A4 phosphate (CA4P) an active constituent that induces tumor necrosis via vascular targeting ${ }^{[62]}$. CA4P is structurally identical to colchicine and binds to tubulin, leading to tubulin depolymerization. CA4P initiates the destabilization of tubulin cytoskeleton; thus interfering with the cyclic formation of microtubule cytoskeleton. CA4P provokes dose- and time-dependent cytotoxic effects on vascular endothelial cells. The tumor necrosis characteristics of $\mathrm{CA} 4 \mathrm{P}$ are greatly assigned to augmented permeability of vascular endothelium, reduction or prohibition of blood flow ${ }^{[63]}$ and apoptosis of endothelial cell ${ }^{[64]}$. CA4P indicated an effective therapeutic advantages in thyroid caricinoma including anaplastic $^{[65]}$ and medullary malignancies ${ }^{[66]}$. Cardiac and vascular damage induced by combretastatin/ CA4P has been shown to occur due to failure of blood circulation within the myocardium. Besides a direct cardiotoxic influence of CA4P on cardiomyocytes has been reported ${ }^{[67]}$. To date 2 vignette cases has been published reporting SCM induced by combretastatin. The reported cases included postmenopausal women (71-78 y) suffering from anaplastic thyroid carcinoma with no previous CAD history ${ }^{[68]}$. Both the patients received combretastatin as an ingredient of primary chemotherapy regimen together with cisplatin and doxorubicin. Following the combretastatin administration; SCM occurred temporarily during the first round. Clinical manifestations included ECG abnormalities (ECG alterations of variable degree), reduced LVEF (interquartile range 40-50 \%), however the symptom of angina was not existent. Coronary angiogram in a single patient suggested paucity of CAD. LV systolic impairment was clearly identified with one patient signifying apical akinesis and apical septal hypokinesis being diagnosed in the second patient. Both the patients indicated absolute restoration of myocardial action together LVEF normalization within a month. Combretastatin-induced SCM patients present either raised LVEF or no noticeable symptoms of SCM in a case a chemotherapeutic regimen involved the simultaneous administration of doxorubicin and combretastatin.

\section{Rituximab:}

Rituximab is a well-recognized monoclonal antibody targeting CD20 antigen on B-cells inclusive of both the normal and cancer cells. Rituximab encompasses a broad range of applications, including its utilization in autoimmune diseases up to the management of hematologic illnesses. It is widely prescribed for the treatment of autoimmune illnesses (rheumatoid arthritis), chronic lymphocytic leukemia (CLL) and follicular nonHodgkin's lymphoma. Moreover its applications as immunosuppressive agent after organ transplantation are also well established. A case of CLL patient (male, $66 \mathrm{y}$ ) that presented dyspnea and flushing after receiving rituximab infusion was reported by $\mathrm{Ng}$ et al. ${ }^{[69]}$. Although the patient indicated no noticeable SCM symptoms however, ECG illustrated ST segment elevation and raised troponin level. Coronary angiogram suggested no expressive CAD. Echocardiographic examination revealed reduced LVEF of $40 \%$ coupled with abnormal wall motion signifying rituximabinduced SCM. Information regarding LVEF recovery was not reported because of patient death from a noncardiac cause. Cardiotoxic side effects reported to be associated with rituximab therapy included cardiac arrhythmias and rarely MI. Yet, the complete profile/ mechanism of rituximab related cardiotoxicity is not well understood ${ }^{[32]}$. Two pathways describing the possible pathophysiology of rituximab-associated SCM have been postulated. Coronary vasospasm caused due to sympathetic stimulation mediated from the activation of neurohormonal system and ventricular dysfunction occurring due to the direct effect of rituximab on cardiac functioning ${ }^{[32]}$. Long-term therapy with rituximab may elevate transforming growth factors (TGF) $-\beta$ along with the diffusion/deposition of reticulin fibers within cardiomyocytes. Thus the rituximab-induced cardiotoxic effects usually appear during the later 
cycles of rituximab infusions; probably due to the deposition of reticulin fibers within cardiomyocytes with the passage of time ${ }^{[70]}$.

\section{Tyrosine kinase inhibitors:}

Tyrosine kinases belong to the class of enzymes accelerating cellular activities after being mediated by external or internal stimuli. Binding of tyrosine kinases to various growth factor receptors present on cytoplasmic domains initiate a signalling cascade, thus provoking cellular regulation. Downstream hyperactivation associated with the mutation or overexpression of theses enzymes may lead to abnormal and excessive overgrowth of cells. Based on the pivotal role of tyrosine kinases in cancer progression, tyrosine kinases may act as an eminent target for chemotherapeutic agent ${ }^{[71]}$. Axitinib is a second generation, tyrosine kinase inhibitor (TKI). It is an orally administered drug preventing the growth of cancer cells via inhibition of angiogenesis, a fundamental event in the growth and proliferation of cancer cells. VEGF receptors, such as VEGFR-1, VEGFR-2, and VEGFR-3 are the potential targets for axitinib ${ }^{[72]}$. The antiproliferative and anticancer activity of sunitinib is mediated via inhibition of multiple receptor tyrosine kinases, involving colony stimulating factor type 1 (CSF-1R), FMS-like tyrosine kinase-3 (FLT3), glial cell-line derived neurotrophic factor receptor (RET), platelet-derived growth factors (PDGFR $\alpha$ and PDGFR $\beta$ ), and VEGFR1, VEGFR2 and VEGFR $3^{[73]}$. The above mentioned chemotherapeutic moieties have been approved for the management of progressive renal cell carcinoma (RCC). Hypertension, hypertensive crisis and thromboembolism are the cardiotoxic effects associated with TKIs. Recently TKIs-induced SCM has been described. Numico et al. reported a case of $57 \mathrm{y}$ old woman with progressive RCC, whounderwent treatment with sunitinib. During the first cycle of treatment, the patient presented clinical features suggestive of SCM, chest pain, ECG abnormalities, lowered LVEF (15-20\%) and nonappearance of significant CAD. The LVEF of patient recovered to the normal range after $3 \mathrm{mo}^{[74]}$. Similarly Ovaida et al., reported SCM-induced with axitinib. The reported case was a female patient (71 y) with progressive metastatic RCC taking axitinib therapy. The patient exhibited SCM features just within $24 \mathrm{~h}$ of drug administration. The clinical manifestations included chest pain and ST segment elevation on the anterior, lowered LVEF (20-25 \%) and normal coronary angiogram (obstructive CAD not existent). LVEF of the patient recovered to the normal range after 3 $\mathrm{w}^{[33]}$. Ibrutinib; a Bruton's tyrosine kinase inhibitor is extensively used for the management of B cell tumors including chronic lymphocytic leukemia, mantle cell lymphoma, and Waldenstrom's macroglobulinemia. Ibrutinib-induced SCM has been recently reported by Gize et al. A reported case was a $53 \mathrm{y}$ old female undertaking ibrutinib treatment for the management of non-small-cell lung cancer. Clinical presentations included ECG changes and LV dysfunction coupled with apical hyperkinesis and mid-ventricular hypokinesis ${ }^{[75]}$.

\section{Immune checkpoint inhibitors:}

A normal immune system of the body is modified by immune checkpoint inhibitors in such a way that the immune checkpoints present over the surface of T-cells are blocked leading to the death of cancer cells. Immune checkpoints are the component of a normal immunoregulatory system. Activation of these checkpoints produces off signal for the T-cells thus preventing the immune system to kill the normal body cells or in case of tumor, inhibits T-cells mediated apoptosis of cancer cells. Binding of proteins allocated over the surface of T-cells with the partner proteins (located on other cells/tumor cells) leads to the generation of off signal. Immune checkpoint inhibitors prevent the binding of T-cells proteins with partner proteins thus initiating the death of cancer cells by the host immune cells/T-cells. Chemotherapeutic moieties included in this class are ipilimumab, pembrolizumab and nivolumab are human antibodies targeted against T lymphocyte antigen-4 (CTLA-4) and programmed death-1 (PD-1 $)^{[76]}$. Introduction of such form of chemotherapy transformed the treatment mode of melanomas $^{[77]}$ and few solid tumors ${ }^{[34]}$. Immune checkpoint inhibitors in spite of their notable applications exhibit some distinctive type of adverse effects termed as immune-related adverse events (irAEs) ${ }^{[35]}$. These adverse effects may cover a wide range including dermatologic, endocrine, gastrointestinal, hepatic, and less frequently inflammatory outcomes. It has been reported that myocardial inflammation is mediated from immune checkpoint inhibitors therapy and in few cases may lead to end stage heart failure ${ }^{[36]}$. Till now a single case has been reported that showed stress syndrome like symptoms such as apical ballooning, hyperdynamic basal segment coupled with normal angiogram suggesting the absence of obstructive CAD. The reported case was a metastatic melanoma patient receiving ipilimumab treatment ${ }^{[78]}$. Obviously the patient indicated noticeable symptoms of myocardial inflammation or myocarditis. 


\section{Other drugs:}

A case of SCM induced by trastuzumab treatment has been reported by Khanji et al. ${ }^{[37]}$. The patient was a 55 y old postmenopausal woman. She was diagnosed with invasive ductal carcinoma of breast and underwent therapy with trastuzumab, docetaxel, and carboplatin. Anginal chest pain, ECG abnormalities and raised troponin contents were noticed during the 11th round of drug administration. Ventriculography of the patient identified decreased LVEF along with mid LV dyskinesis and normal apical and basal wall motions, the condition being suggestive of reverse stress (atypical variant SCM). Coronary angiography produced normal coronary angiogram. The LV dysfunction recovery occurred in a month. Type II chemotherapy-related cardiotoxicity is associated with trastuzumab therapy and is reported to occur in 3.2 to $18.6 \%$ of cases with major clinical manifestation of reversible ventricular dysfunction ${ }^{[56]}$. Cardiotoxicity induced by trastuzumab is demonstrated by LV dysfunction imparting minor interference to the normal growth, maintenance and regeneration of cardiac myocytes. The variation among the clinical manifestations and pathogenesis of trastuzumabinduced cardiotoxic effects (ventricular dysfunction) and SCM is still not clear. Moreover reintroduction of trastuzumab therapy after normalization of LVEF is the subject that requires additional consideration. Goel et al. reported a daunorubicin-related SCM case. The reported case was a $55 \mathrm{y}$ male patient with non-M3 acute myeloid leukemia (AML) and received daunorubicin and cytarabine therapy. SCM features appeared after 6 $\mathrm{d}$ of the administration of therapy ${ }^{[79]}$. Mitsumori et al. also reported SCM caused by daunorubicin in a $67 \mathrm{y}$ female diagnosed with refractory multiple myeloma ${ }^{[80]}$. Anthracyclines are one of most famous antineoplastic agents used for the management of blood cancers and solid tumors. The mechanisms engaged behind the occurrence of anthracyclines-mediated cardiotoxicity included, cardiac side effects resulting from the breakage of DNA double-strand, ROS (reactive oxygen species) generation and mitochondrial dysfunction ${ }^{[81]}$. Inevitable harm to cardiac myocytes occurs due to malfunctioning of mitochondria and deficiency of antioxidant enzymes thus causing Type I chemotherapyrelated cardiotoxicity ${ }^{[82]}$. The factor needs to be explored is whether anthracyclines therapy induces reversible cardiomyopathy/stress syndrome.

Twenty five cases of SCM-induced by chemotherapy have been covered and discussed in the current review. On the average 59 y age (14-83 y) was perceived in all the reviewed patients. Despite the fact that in general cases postmenopausal women are primarily affected from SCM however, the current review explored that patient of both sexes presented equal incidence of chemotherapy-induced SCM. Earlier CAD history being not recognized in any of the reported patients. As per this review; majority of patients indicated a clinical manifestation of chest pain (54\%). ST segment irregularities being noted in $75 \%$ patients while rest of the patients presented $\mathrm{T}$ wave changes during investigation. Extremely reduced LVEF was diagnosed in all patients with approximately $27 \%$ mean value (normal range 55-75\%). The entire patient assessed in a current literature presented LVEF normalization/ recovery in an average period of $1 \mathrm{mo}(5 \mathrm{~d}$ to $3 \mathrm{mo}$ duration). The attained response suggests the incidence of reversible LVEF and thus an adequate therapeutic effect. Effective prognosis was perceived that was greatly supported by the fact that among all the treated patients' only 3 patients died. Among these 3 patients only one patient died of cardiotoxicity while the death of the other 2 patients occurred from a non-cardiac cause (death of one patient was attributed to endstage tumor progression whereas pathological fracture caused the death of the other patient). Advanced therapies, such as intra-aortic balloon pump (IABP) or mechanical circulatory support has not been utilized in any patient except 2 patients were facilitated with IABP. This review recommended the association of a wide spectrum of antineoplastic agents with SCM induction and the information being compatible with previously reported literature. Numerous drugs including 5-FU (42 $\%$ ) capecitabine (21\%), bevacizumab, combretastatin, daunorubicin, ipilimumab, rituximab, trastuzumab and tyrosine kinase inhibitors such as axitinib, sunitinib, and ibrutinib were found to initiate the stress syndrome. Majority of the reviewed cases suggested the occurrence of SCM during the first round of chemotherapy ( $68 \%$; 17 out of 25 cases). Generally the chemotherapeutic dose used for the management of reported cases has been poorly defined therefore, a correlation among the therapeutic dose and SCM was difficult to arrive at. According to the findings of a retrospective study performed at the MD Anderson Cancer Center, the cancers most frequently associated with the SCM induction include lymphoproliferative neoplasms (23.3 $\%$ ), followed by gastrointestinal (17\%), ovarian (13.3 $\%)$ and breast cancer $(10 \%)^{[75]}$. However the presnet review suggested the prevalence of chemotherapyinduced SCM in patients of breast cancers, head and neck, and gastrointestinal malignancies. The prominent 
risk of SCM occurrence in cancer patients is related to emotional and psychological stress (developed due to the diagnosis of cancer or its particular stage in an individual) biochemical stress (developed from the systemic treatment with numerous antineoplastic agents) and physical stress (related to the surgical or radiation therapy of cancer). The actual reason involved behind the incidence of SCM in cancer patients treated with chemotherapy is undiscovered. However the assessment of hospitalized patients using the National Inpatient Sample database (2010 to 2014) indicated increased occurrence of SCM in patients getting antineoplastic therapy (overall or nationwide trend) ${ }^{[83]}$. Not only the risk of SCM occurrence is raised in cancer patients; these patients exhibited extremely worse aftereffects as compared to the SCM patients without cancer malignancy. According to the findings established from National Inpatient Sample (NIS) analysis "SCM patients with cancer malignancy present mortality rate (13.8 vs. $2.9 \%, \mathrm{p}<0.0001$ ), duration of hospital stay ( 7 vs. $4 \mathrm{~d}, \mathrm{p}<0.0001$ ) and overall treatment expenses (\$29 291 vs. \$36 231, p<0.0001) significantly higher than SCM patients without cancer disease ${ }^{[84]}$. Hence the earlier diagnosis and immediate provision of suitable therapy in cancer patients may help to reduce the mortality rate and overall treatment expenses.

Based on the typical guideline directed medical therapy (GDMT), angiotensin converting enzyme (ACE) inhibitors, angiotensin II receptor blockers, $\beta$-blockers and diuretics (to control excessive volume) are prescribed for the management of heart failure along with lowered LVEF in hemodynamically stable SCM patients ${ }^{[85]}$. Extra care together with vasodilator therapy being required in obstructive left ventricular outflow tract (LVOT) to prevent volume reduction. Additionally, inotropic drugs, such as dopamine and dobutamine could not be used for the management of LVOT related hypotension since obstruction degree is further exaggerated with these medication ${ }^{[38]}$. Preliminary clinical trials/investigations based information is unavailable to define the optimum duration of medical treatment in SCM subjects. Generally, GDMT is prolonged till the normalization of systolic function (LVEF) that usually takes duration of $1-4 \mathrm{w}^{[86]}$. The information regarding the discontinuation of GDMT after the recovery of LVEF is not well understood. Recently a TRED-HF study design (pilot clinical trial study), investigated the outcome of therapy withdrawal in patients with past history of dilated cardiomyopathy (DCM) that indicated complete recovery of their LVEF however, greatly supported the significance of medical treatment continuation for an undefined period ${ }^{[87]}$. Findings of a current clinical study indicated that treatment withdrawal caused revert of cardiomyopathy in about $44 \%$ of patients during initial 6 mo. It is currently recommended to continue medical therapy in DCM patients for an unspecified duration based on the elevated relapse rate perceived in these patients. Similarly in SCM patients; same criteria of continued medical treatment for an indefinite duration is preferred based on the relapse rate of about $1.5 \%{ }^{[88]}$. Observational study revealed that the administration of ACE inhibitors improved the survival of patients for about $1 \mathrm{y}^{[88]}$. Optimum therapy required for the management of SCM patients' needs to be clearly explored with the assistance of supplementary preliminary clinical trial studies.

Reintroduction of therapy with the cardiotoxic anticancer drug presents significant clinical challenges in patients requiring continuous cancer treatment. Limited information is available regarding the safety profile of particular antineoplastic drug upon reinstatement. In a retrospective study conducted on 30 cancer induced SCM patients taking therapy at MD Anderson Cancer Centre, 21 candidates needed persistent therapy with antineoplastic agents. Sixteen out of 21 patients were capable of securely resume chemotherapy after LVEF restoration without relapse of SCM. Following SCM, cancer therapy was recommenced in $20 \mathrm{~d}^{[76]}$. Among the 25 reported cases reviewed in current literature, 2 patients underwent re-establishment of 5-FU-based chemotherapy. One of the patients tolerated half of the therapeutic dose (previously administered) and the patient faced cardiac arrest when the dose was increased to maximum level ${ }^{[18]}$. In the second candidate cardiac arrest occurred after the administration of maximum therapeutic dose up to 4 rounds $^{[44]}$. Thus reintroduction of chemotherapeutic agent in SCM cancer patients required further investigation. Current review is unable to answer the following two important queries $^{[1]}$ : whether SCM or any other disease (such as chemotherapy-associated myocarditis or chemotherapyinduced reversible non-ischemic cardiomyopathy) was presented by the reported cases reviewed? ${ }^{[2]}$ If the reported cases truly presented SCM then was the chemotherapeutic moiety the main causative agent responsible for SCM induction? Patients displaying only chest pain or dyspnea symptoms during or after the administration of anticancer drugs may be diagnosed with various cardiac disorders including acute coronary syndrome, cardiac side effects mediated cardiac failure, myocarditis, stress syndrome and venous 
thromboembolism.

Markers of cardiac necrosis, echocardiography, coronary angiography (to prevent acute coronary plaque rupture), CT pulmonary angiogram (to prevent pulmonary thromboembolism), natriuretic peptides, cardiac MRI and myocardial biopsy (to prevent myocarditis) are the numerous diagnostic tests used for earlier detection of cardiac dysfunction. Clinical features including LV wall motion deformities of apical and mid sections that expand beyond coronary distribution and the characteristic apical ballooning occurrence designates the diagnosis of SCM. However, in case of patients presenting a diffused or global form of LV contractile dysfunction; the chemotherapy-induced SCM is hardly distinguished from alternative chemotherapy-induced cardiac effects. Internationally no exact description of cardiotixicity is available; however asymptomatic reduction in LVEF $(\geq 10 \%)$ beyond a lower limit (normal range 50 to $55 \%$ ) is the description widely accepted by most of the organization. Nevertheless during the existence of clinical manifestations of cardiac failure; cardiac toxicity demonstrates no significant decline in $\mathrm{LVEF}^{[89,90]}$. In cancer patients several factors apart from chemotherapy may contribute to SCM incidence such as emotional or psychological tension associated with cancer identification or recognition of its particular inflammatory state, physical stress associated with surgical or radiotherapy mediated cancer management. The trigger mainly responsible for SCM induction is difficult to identify and to date no study clearly concludes the antineoplastic drugs as the chief triggers responsible for SCM induction. Further investigation is required to explore the pivotal role of chemotherapeutic agents in SCM induction along with the discovery of novel targeted moieties acting as a potential triggers for induction of stress syndrome.

Increased risk of SCM incidence has been noticed in cancer patients taking multiple sorts of therapies for the management of their illnesses including surgical treatment, radiotherapy and chemotherapy. In comparison to the general population, remarkably worse consequences are presented by chemotherapyinduced SCM patients therefore on time recognition, prolonged monitoring and appropriate treatment of such patients is extremely desirable. Although SCM incidence is easily identified in cancer patients however, it interrupts chemotherapeutic management that ultimately augment the after effects of cancer. The factor needs to be further explored is whether cardiomyopathy induced by chemotherapy characterizes
$\mathrm{SCM}$ in real or an alternative chemotherapy related reversible cardiac toxicity. Further if the chemotherapy related cardiomyopathy in SCM is real, subsequently an important subject that needs to be clarified in this scenario is whether the chemotherapeutic drug is the definite cause of stress syndrome/SCM?

\section{Conflict of interest:}

All authors report no conflicts of interest in this work.

\section{REFERENCES}

1. Dote K, Sato H, Tateishi H, Uchida T, Ishihara M. Myocardial stunning due to simultaneous multivessel coronary spasms: a review of 5 cases. J Cardiol 199;21:203-14.

2. Wittstein IS, Thiemann DR, Lima JAC, Baughman KL, Schulman SP, Gerstenblith G, et al. Neurohumoral features of myocardial stunning due to sudden emotional stress. N Engl J Med 2005;352:539-48.

3. Sharkey SW, Windenburg DC, Lesser JR, Maron MS, Hauser $\mathrm{RG}$, Lesser JN, et al. Natural history and expansive clinical profile of stress (tako-tsubo) cardiomyopathy. J Am Coll Cardiol 2010;55:333-41.

4. Kumar G, Holmes DR, Prasad A. "Familial" apical ballooning syndrome (Takotsubo cardiomyopathy). Int $\mathrm{J}$ Cardiol 2010;144:444-5.

5. Dib C, Asirvatham S, Elesber A, Rihal C, Friedman P, Prasad A. Clinical correlates and prognostic significance of electrocardiographic abnormalities in apical ballooning syndrome. Am Heart J 2009;157:933-8.

6. Ito K, SUGiHARA H, Kawasaki T, Yuba T, Doue T, Tanabe $\mathrm{T}$, et al. Assessment of ampulla (Takotsubo) cardiomyopathy with coronary angiography, two dimensional echocardiography and $99 \mathrm{~m}$ Tc-tetrofosmin myocardial single photon emission computed tomography. Ann Nucl Med 2001;15:351-5.

7. Gianni M, Dentali F, Grandi AM, Sumner G, Hiralal R, Lonn E. Apical ballooning syndrome or takotsubo cardiomyopathy: a systematic review. Eur Heart J 2006;27:1523-9.

8. Bybee KA, Prasad A. Stress-related cardiomyopathy syndromes. Circulation 2008;118:397-409.

9. Lee PH, Song JK, Sun BJ, Choi HO, Seo JS, Na JO, et al. Outcomes of patients with stress-induced cardiomyopathy diagnosed by echocardiography in a tertiary referral hospital. J Am Soc Echocardiogr 2010;23:766-71.

10.Abraham J, Mudd JO, Kapur N, Klein K, Champion HC, Wittstein IS. Stress cardiomyopathy after intravenous administration of catecholamines and beta-receptor agonists. J Am Coll Cardiol 2009;53:1320-5.

11.Amariles P, Cifuentes L. Drugs as Possible Triggers of Takotsubo Cardiomyopathy: A comprehensive literature search updates 2015. Curr Clin Pharmacol 2016;11:95-109.

12.Madias JE. What is/are the trigger(s) of takotsubo syndrome in cancer patients receiving chemotherapy? Int J Cardiol 2016;222:1.

13.De Pasquale MD, Mastronuzzi A, De Sio L, Serra A, Grimaldi C, Chinali M, et al. Transient global ventricular dysfunction in an 
adolescent affected by pancreatic adenocarcinoma. BMC Pediatr 2016;16:99.

14. Volkova M, Russell IR. Anthracycline cardiotoxicity: prevalence, pathogenesis and treatment. Curr Cardiol Rev 2011;7:214-20.

15.Ewer MS, Ewer SM. Cardiotoxicity of anticancer treatments. Nat Rev Cardiol. 2015;12:620.

16.Curigliano G, Cardinale D, Dent S, Criscitiello C, Aseyev O, Lenihan $\mathrm{D}$, et al. Cardiotoxicity of anticancer treatments: epidemiology, detection, and management. CA Cancer J Clin 2016;66:309-25.

17.Yeh ET, Tong AT, Lenihan DJ, Yusuf SW, Swafford J, Champion $\mathrm{C}$, et al. Cardiovascular complications of cancer therapy: diagnosis, pathogenesis, and management. Circulation 2004;109(:3122-31.

18.Basselin C, Fontanges T, Descotes J, Chevalier P, Bui-Xuan B, Feinard G, et al. 5fluorouracil-induced Tako-Tsubo-like syndrome. Pharmacotherapy 2011;31:226.

19. Naranjo CA, Busto U, Sellers EM, Sandor P, Ruiz I, Roberts E, et al. A method for estimating the probability of adverse drug reactions. Clin Pharmacol Ther 1981;30:239-45.

20.Stewart T, Pavlakis N, Ward M. Cardiotoxicity with 5-fluorouracil and capecitabine: more than just vasospastic angina. Intern Med J 2010;40:303-7.

21.Dalzell JR, Samuel LM. The spectrum of 5-fluorouracil cardiotoxicity. AntiCancer Drugs 2009;20:79-80.

22.Parker WB, Cheng YC. Metabolism and mechanism of action of 5 fluorouracil. Pharmacol Ther 1990;48:381-95.

23. Ozturk MA, Ozveren O, Cinar V, Erdik B, Oyan B. Takotsubo syndrome: an underdiagnosed complication of 5-fluorouracil mimicking acute myocardial infarction. Blood Coagul Fibrinolysis 2013;24:90-4.

24.Becker K, Erckenbrecht JF, Häussinger D, Fueling T. Cardiotoxicity of the antiprolif erative compound fluorouracil. Drugs 1999;57:475-84.

25.Tsibiribi P, Descotes J, Lombard-Bohas C, Barel C, Bui-Xuan $\mathrm{B}$, Belkhiria M, et al. Cardiotoxicity of 5-fluorouracil in 1350 patients with no prior history of heart disease. Bull Cancer 2006;93:10027-30.

26.Kobayashi N, Hata N, Yokoyama S, Shinada T, Shirakabe A, Mizuno K. A case of Takotsubo cardiomyopathy during 5-fluorouracil treatment for rectal adenocarcinoma. J Nippon Med Sch 2009;76:27-33.

27.Qasem A, Abdulhak AAB, Aly A, Moormeier J. Capecitabineinduced takotsubo cardiomyopathy: a case report and literature review. Am J Ther 2016;23:e1188-e92.

28. Y-Hassan S. Global Takotsubo syndrome and cardiogenic shock: case report. Reactions 2013;1441:2.

29.Endo A, Yoshida Y, Nakashima R, Takahashi N, Tanabe K. Capecitabine induces both cardiomyopathy and multifocal cerebral leukoencephalopathy. Int Heart J 2013;54:417-20.

30.Molteni LP, Rampinelli I, Cergnul M, Scaglietti U, Paino AM, Noonan DM, et al. Capecitabine in breast cancer: the issue of cardiotoxicity during fluoropyrimidine treatment. Breast $\mathrm{J}$ 2010;16:S45-8.

31.Izumiya Y, Shiojima I, Sato K, Sawyer DB, Colucci WS, Walsh
K. Vascular endothelial growth factor blockade promotes the transition from compensatory cardiac hypertrophy to failure in response to pressure overload. Hypertension 2006;47:887-93.

32.Cheungpasitporn W, Kopecky SL, Specks U, Bharucha K, Fervenza FC. Nonischemic cardiomyopathy after rituximab treatment for membranous nephropathy. J Renal Inj Prev 2017;6:18-25.

33.Ovadia D, Esquenazi Y, Bucay M, Bachier CR. Association between takotsubo cardiomyopathy and axitinib: case report and review of the literature. J Clin Oncol 2014;33:e1-3.

34.Garber K. Driving T-cell immunotherapy to solid tumors. Nat Biotechnol 2018;36:215-9.

35.Champiat S, Lambotte O, Barreau E, Belkhir R, Berdelou A, Carbonnel F, et al. Management of immune checkpoint blockade dysimmune toxicities: a collaborative position paper. Ann Oncol 2016;27:559-74.

36. Norwood TG, Westbrook BC, Johnson DB, Litovsky SH, Terry NL, McKee SB, et al. Smoldering myocarditis following immune checkpoint blockade. J Immunother Cancer 2017;5:91.

37.Khanji M, Nolan S, Gwynne S, Pudney D, Ionescu A. TakoTsubo syndrome after trastuzumab an unusual complication of chemotherapy for breast cancer. Clin Oncol 2013;25:329.

38.Villareal RP, Achari A, Wilansky S, Wilson JM. Anteroapical stunning and left ventricular outflow tract obstruction. Mayo Clin Proc 2001;76:79-83.

39.Heinemann V, von Weikersthal LF, Decker T, Kiani A, VehlingKaiser U, AlBatran SE, et al. FOLFIRI plus cetuximab versus FOLFIRI plus bevacizumab as first-line treatment for patients with metastatic colorectal cancer (FIRE-3): a randomised, openlabel, phase 3 trial. Lancet Oncol 2014;15:1065-75.

40.Goldberg RM, Sargent DJ, Morton RF, Fuchs CS, Ramanathan RK, Williamson SK, et al. Randomized controlled trial of reduced-dose bolus fluorouracil plus leucovorin and irinotecan or infused fluorouracil plus leucovorin and oxaliplatin in patients with previously untreated metastatic colorectal cancer: a north American intergroup trial. J Clin Oncol 2006;24:3347-53.

41.Labianca R, Beretta G, Clerici M, Fraschini P, Luporini G. Cardiac toxicity of 5fluorouracil: a study on 1083 patients. Tumori J 1982;68:505-10.

42. Cheriparambil KM, Vasireddy H, Kuruvilla A, Gambarin B, Makan M, Saul BI. Acute reversible cardiomyopathy and thromboembolism after cisplatin and 5-fluorouracil chemotherapy: a case report. Angiology 2000;51:873-8.

43. Grunwald MR, Howie L, Diaz LA Jr. Takotsubo cardiomyopathy and fluorouracil: case report and review of the literature. J Clin Oncol 2011;30:e11-e4.

44.Radhakrishnan V, Bakhshi S. 5-fluorouracil-induced acute dilated cardiomyopathy in a pediatric patient. J Pediatr Hematol Oncol 2011;33:323.

45.Mosseri M, Chokshi SK, Isner JM. In: Fingert HJ. 5-FU induced vasoconstriction in isolated rabbit aortic rings. Proceedings of the AACR 1990.

46. Robben NC, Pippas AW, Moore JO. The syndrome of 5-fluorouracil cardiotoxicity. An elusive cardiopathy. Cancer 1993;71:493-509.

47.Gianni M, Dentali F, Lonn E. 5 flourouracil-induced apical 
ballooning syndrome: a case report. Blood Coagul Fibrinolysis 2009;20:306-8.

48.Knott K, Starling N, Rasheed S, Foran J, Cafferkey C, Rosen $\mathrm{S}$, et al. A case of Takotsubo syndrome following 5-fluorouracil chemotherapy. Int J Cardiol 2014;177:e65-e7.

49.Saif MW, Shah MM, Shah AR. Fluoropyrimidine-associated cardiotoxicity: revisited. Expert Opin Drug Saf 2009;8:191-202.

50.Segalini G, Labianca R, Beretta G, Borsotti E, Calasso F, Carini $\mathrm{L}$, et al. Cardiotoxicity of 5-fluorouracil. Personal cases and review of the literature. G Ital Cardiol 1987;17:781-5.

51.Ishitsuka H. Capecitabine: preclinical pharmacology studies. Investig New Drugs 2000;18:343-54.

52.Longley DB, Harkin DP, Johnston PG. 5-fluorouracil: mechanisms of action and clinical strategies. Nat Rev Cancer 2003;3:330.

53.Bertolini A, Fiumanò M, Fusco O, Muffatti A, Scarinci A, Pontiggia $\mathrm{G}$, et al. Acute cardiotoxicity during capecitabine treatment: a case report. Tumori J 2001;87:200-6.

54.Jensen SA, Sørensen JB. Risk factors and prevention of cardiotoxicity induced by 5-fluorouracil or capecitabine. Cancer Chemother Pharmacol 2006;58:487-93.

55.Yeh ETH, Bickford CL. Cardiovascular complications of Cancer therapy: incidence, pathogenesis, diagnosis, and management. J Am Coll Cardiol 2009;53:2231-47.

56.Ellis LM. Mechanisms of action of bevacizumab as a component of therapy for metastatic colorectal cancer. Semin Oncol 2006;33(:S1-7.

57.Zachary I, Martin JF, Barnard K. An explanation for the cardiovascular effects of bevacizumab and rofecoxib? Circulation 2006;114:173-5.

58. Sandler A, Gray R, Perry MC, Brahmer J, Schiller JH, Dowlati A, et al. Paclitaxel-carboplatin alone or with bevacizumab for non-small-cell lung cancer. N Engl J Med 2006;355:2542-50.

59. Scappaticci FA, Skillings JR, Holden SN, Gerber H-P, Miller K, Kabbinavar F, et al. Arterial thromboembolic events in patients with metastatic carcinoma treated with chemotherapy and bevacizumab. J Natl Cancer Inst 2007;99:1232-9.

60.Franco TH, Khan A, Joshi V, Thomas B. Takotsubo cardiomyopathy in two men receiving bevacizumab for metastatic cancer. Ther Clin Risk Manag 2008;4:1367-70.

61.Drimal J, Zurova-Nedelcevova J, Knezl V, Sotnikova R, Navarova J. Cardiovascular toxicity of the first line cancer chemotherapeutic agents: doxorubicin, cyclophosphamide, streptozotocin and bevacizumab. Neuro Endocrinol lett 2006;27:176-9.

62.Dziba JM, Marcinek R, Venkataraman G, Robinson JA, Ain KB. Combretastatin A4 phosphate has primary antineoplastic activity against human anaplastic thyroid carcinoma cell lines and xenograft tumors. Thyroid 2002;12:1063-70.

63.Rustin GJ, Galbraith SM, Anderson H, Stratford M, Folkes LK, Sena L, et al. Phase I clinical trial of weekly combretastatin A4 phosphate: clinical and pharmacokinetic results. J Clin Oncol 2003;21:2815-22.

64.Cooney MM, Radivoyevitch T, Dowlati A, Overmoyer B, Levitan N, Robertson K, et al. Cardiovascular safety profile of combretastatin a4 phosphate in a single-dose phase I study in patients with advanced cancer. Clin Cancer Res 2004;10:96-100.

65. Yeung S-CJ, She M, Yang H, Pan J, Sun L, Chaplin D. Combination chemotherapy including combretastatin A4 phosphate and paclitaxel is effective against anaplastic thyroid cancer in a nude mouse xenograft model. J Clin Endocrinol Metab 2007;92:2902-9.

66.Nelkin BD, Ball DW. Combretastatin A-4 and doxorubicin combination treatment is effective in a preclinical model of human medullary thyroid carcinoma. Oncol Rep 2001;8:157217.

67. Tochinai R, Nagata Y, Ando M, Hata C, Suzuki T, Asakawa N, et al. Combretastatin A4 disodium phosphate-induced myocardial injury. J Toxicol Pathol 2016;29:163-71.

68.Bhakta S, Flick SM, Cooney MM, Greskovich JF, Gilkeson RC, Remick SC, et al. Myocardial stunning following combined modality Combretastatin-based chemotherapy: two case reports and review of the literature. Clin Cardiol 2009;32:E80-4.

69.Ng KH, Dearden C, Gruber P. Rituximab-induced Takotsubo syndrome: more cardiotoxic than it appears? BMJ Case Rep 2015;2015:bcr2014208203.

70.Kanamori H, Tsutsumi Y, Mori A, Kawamura T, Obara S, Shimoyama $\mathrm{N}$, et al. Delayed reduction in left ventricular function following treatment of nonHodgkin's lymphoma with chemotherapy and rituximab, unrelated to acute infusion reaction. Cardiology 2006;105:184-7.

71.Workman P, Brunton V, Robins D. Tyrosine kinase inhibitors. Seminars in cancer biology 1992.

72.Rini BI, Melichar B, Ueda T, Grunwald V, Fishman MN, Arranz $\mathrm{JA}$, et al. Axitinib with or without dose titration for first-line metastatic renal-cell carcinoma: a randomised double-blind phase 2 trial. Lancet Oncol 2013;14:1233-42.

73.Motzer RJ, Hutson TE, Tomczak P, Michaelson MD, Bukowski $\mathrm{RM}$, Oudard S, et al. Overall survival and updated results for sunitinib compared with interferon alfa in patients with metastatic renal cell carcinoma. J Clin Oncol 2009;27:3584-90.

74.Numico G, Sicuro M, Silvestris N, Mozzicafreddo A, Trogu A, Malossi A, et al. Takotsubo syndrome in a patient treated with sunitinib for renal cancer. J Clin Oncol 2012;30:e218-e20.

75.Giza DE, Lopez-Mattei J, Vejpongsa P, Munoz E, Iliescu G, Kitkungvan D, et al. Stress-induced cardiomyopathy in Cancer patients. Am J Cardiol 2017;120:2284-8.

76. Weber J. Immune Checkpoint Proteins: A New Therapeutic Paradigm for Cancer 2014; Preclinical Background: CTLA-4 and PD-1 Blockade. Semin Oncol 2010;37:430-9.

77.Lugowska I, Teterycz P, Rutkowski P. Immunotherapy of melanoma. Contemp Oncol 2018;22(:61-7.

78.Geisler BP, Raad RA, Esaian D, Sharon E, Schwartz DR. Apical ballooning and cardiomyopathy in a melanoma patient treated with ipilimumab: a case of takotsubo-like syndrome. J Immunother Cancer 2015;3:4.

79.Goel S, Sharma A, Garg A, Chandra A, Shetty V. Chemotherapy induced Takotsubo cardiomyopathy. World J Clin Cases 2014;2:565.

80.Mitsumori T, Nakajima K, Nozaki Y, Hamanaka S, Nagashima T, Kirito K, et al. Multiple myeloma complicated with Takotsubo cardiomyopathy. Rinsho ketsueki 2010;51:291-6. 
81.Gewirtz D. A critical evaluation of the mechanisms of action proposed for the antitumor effects of the anthracycline antibiotics adriamycin and daunorubicin. Biochem Pharmacol 1999;57:727-41.

82.Goormaghtigh E, Huart P, Praet M, Brasseur R, Ruysschaert J-M. Structure of the adriamycin-cardiolipin complex: role in mitochondrial toxicity. Biophys Chem 1990;35:247-57.

83.Desai R, Abbas SA, Goyal H, Durairaj A, Fong HK, Hung O, et al. Frequency of Takotsubo cardiomyopathy in adult patients receiving chemotherapy (from a 5 y Nationwide inpatient study). Am J Cardiol 2019;123:667-73.

84.Joy PS, Guddati AK, Shapira I. Outcomes of Takotsubo cardiomyopathy in hospitalized cancer patients. J Cancer Res Clin Oncol 2018;144:1539-45.

85.Bybee KA, Kara T, Prasad A, Lerman A, Barsness GW, Wright RS, et al. Systematic review: transient left ventricular apical ballooning: a syndrome that mimics ST-segment elevation myocardial infarction. Ann Intern Med 2004;141:858-65.

86. Sharkey SW, Lesser JR, Zenovich AG, Maron MS, Lindberg $\mathrm{J}$, Longe $\mathrm{TF}$, et al. Acute and reversible cardiomyopathy provoked by stress in women from the United States. Circulation 2005;111:472-9.

87.Halliday BP, Wassall R, Lota AS, Khalique Z, Gregson J, Newsome $\mathrm{S}$, et al. Withdrawal of pharmacological treatment for heart failure in patients with recovered dilated cardiomyopathy (TRED-HF): an open-label, pilot, randomised trial. Lancet 2019;393:61-73.

88.Singh K, Carson K, Usmani Z, Sawhney G, Shah R, Horowitz J. Systematic review and meta-analysis of incidence and correlates of recurrence of takotsubo cardiomyopathy. Int $\mathrm{J}$ Cardiol 2014;174: 696-701.

89.Seidman A, Hudis C, Pierri MK, Shak S, Paton V, Ashby M, et al. Cardiac dysfunction in the trastuzumab clinical trials experience. J Clin Oncol 2002; 20:1215-21.

90.Plana JC, Galderisi M, Barac A, Ewer MS, Ky B, ScherrerCrosbie M, et al. Expert consensus for multimodality imaging evaluation of adult patients during and after cancer therapy: a report from the American Society of Echocardiography and the European Association of Cardiovascular Imaging. Eur Heart J Cardiovasc Imaging 2014;15:1063-93.

This is an open access article distributed under the terms of the Creative Commons Attribution-NonCommercial-ShareAlike 3.0 License, which allows others to remix, tweak, and build upon the work non-commercially, as long as the author is credited and the new creations are licensed under the identical terms

This article was originally published in a special
issue, "Biomedical Research in Healthcare Setting"
Indian J Pharm Sci 2020:82(2)Spl issue5;27-40

